\title{
Surface energy of free clusters of bubbles: an estimation
}

\author{
M. F. Vaz†, M. A. Fortes \\ Instituto de Ciência e Engenharia de Superfícies, \\ Departamento de Engenharia de Materiais, Instituto Superior Técnico, \\ Avenda Rovisco Pais, 1049-001, Lisboa, Portugal \\ and F. Graner
}

CNRS Unitée Mixte ássociée au 5588 et Université Grenoble I, Laboratoire de Spectrométrie Physique, BP 87, 38402 St Martin d'Hères Cedex, France

[Received in final form 19 June 2002 and accepted 8 July 2002]

\section{A BSTRACT}

We propose an approximate equation for the surface energy of twodimensional free bubble clusters which we compare with exact calculations of the surface energy of symmetrical clusters consisting of a central bubble surrounded by one or two shells of bubbles of two different areas. The accuracy of the equation is good for relatively narrow distributions of the areas and of the number of sides of the bubbles but underestimates the energy for large widths of those distributions. We propose a similar approximate equation for the surface energy of three-dimensional clusters.

The energy of foam clusters with an incompressible gas reduces to its surface energy $E=P \gamma$, where $P$ is the total length (in two-dimensional (2D) clusters) or area (in three-dimensional (3D) clusters) of the films separating adjacent bubbles; $\gamma$ is the film tension. The energy depends on the strain in the cluster; only free clusters will be considered in this letter. Different arrangements (topologies) of a large number of bubbles of given sizes (area or volume) do not seem to affect the energy very much (Weaire and Phelan 1994, Graner et al. 2001, Vaz and Fortes 2001). The metastable arrangements have an energy that is close to that of the absolute minimum energy arrangement. In small clusters, however, the energy may be more sensitive to topology (Weaire and Phelan 1994, Graner et al. 2001, Vaz and Fortes 2001) since the peripheral bubbles have relatively larger energies; the shape of the cluster is important and round clusters tend to have smaller energy.

This small sensitivity of surface energy as regards topology indicates that it should be possible to estimate the energy from the areas (in two dimensions) or volumes (in three dimensions) of the bubbles, at least for clusters with a fairly large number of bubbles. Along these lines, Graner et al. (2001) proposed in a recent paper the following approximate equation for the surface energy $E=P \gamma$ of a large 2D free cluster of bubbles, such that the fraction of peripheral bubbles relative to the total number is negligible:

\footnotetext{
† Author for correspondence. Email: fatima.vaz@ist.utl.pt.

Philosophical Magazine Letters ISSN 0950-0839 print/ISSN 1362-3036 online (C) 2002 Taylor \& Francis Ltd 


$$
\frac{E}{\gamma} \approx \frac{3.722}{2} \sum_{i} A_{i}^{1 / 2}
$$

where $A_{i}$ is the area of bubble $i$ and the sum includes all bubbles in the cluster. The factor 3.722 is the perimeter of a regular hexagon of area 1; the figure for regular Plateau cells with $n$ sides (i.e. cells bounded by $n$ identical circular films meeting at $120^{\circ}$ in vertices) is weakly dependent on $n$, decreasing from 3.742 for $n=3$ to 3.712 for $n=\infty$ (Graner et al. 2001).

Equation (1) is exact for a (regular) honeycomb; deviations are expected to increase as the widths of the distributions of cell area and number of sides increase (Graner et al. 2001). In fact, equation (1) should better be regarded as an approximate lower bound to the energy of a large cluster. Note that the honeycomb is the minimum perimeter partition of the plane into regions of equal area (Hales 2001). When partitions into regions of two different areas are considered, the factor in equation (1) can be reduced to (3.692/2) (Teixeira et al. 2002). This is likely to be a lower bound for $P / \sum_{i} A_{i}^{1 / 2}$ in unbounded clusters.

For finite clusters, the additional contribution of the cluster external boundary to the energy has to be considered. In this letter we take this contribution into account and propose an approximate equation for the total energy of arbitrary tree clusters. The cluster boundary energy term will be written in the form

$$
E_{\mathrm{b}}={ }_{S_{\mathrm{b}} \gamma_{\mathrm{b}}},
$$

where $s_{\mathrm{b}}$ is the (macroscopic) perimeter of the boundary and $\gamma_{\mathrm{b}}$ is an average specific (free) energy associated with the cluster boundary. In other words, $\gamma_{b}$ is the surface tension of the cluster, regarded as a homogeneous fluid. The boundary energy $\gamma_{\mathrm{b}}$ was recently estimated by Fortes and Rosa (2001) for a monodisperse cluster of hexagonal bubbles. Using a broken bond approach, they obtained

$$
\gamma_{\mathrm{b}}=0.576 \gamma
$$

independent of bubble size, for the average (over orientation) external boundary energy of a honeycomb. $\gamma_{b}$ can in fact vary between $0.522 \gamma$ and $0.604 \gamma$, the former applying to a boundary parallel to a close-packed row of hexagons. We shall use the average $\gamma_{\mathrm{b}}$ (equation (3)) for arbitrary clusters and assume that $\gamma_{\mathrm{b}}$ is little affected by topology. For a round (equiaxed) cluster we take

$$
s_{\mathrm{b}}=2\left(\pi \sum_{i} A_{i}\right)^{1 / 2} \text {. }
$$

We therefore suggest the following equation to estimate the surface energy of a round $2 \mathrm{D}$ cluster

$$
\frac{E}{\gamma}=\frac{3.722}{2} \sum_{i} A_{i}^{1 / 2}+2.042\left(\sum_{i} A_{i}\right)^{1 / 2} .
$$

It should be noted that, for a cluster of regular hexagons of the same area and with a regular hexagonal boundary along close-packed rows, the factor 2.042 in equation (5) decreases to $3.722 \times 0.522=1.943$ (3.722 is the ratio of the perimeter to the square root of the area of a regular hexagon). Combining this with the lower bound $(3.692 / 2)$ for an unbounded cluster leads to a tentative lower bound for the energy of a large finite $2 \mathrm{D}$ cluster: 


$$
\left(\frac{E}{\gamma}\right)_{1 \mathrm{~b}}=\frac{3.692}{2} \sum_{i} A_{i}^{1 / 2}+1.943\left(\sum_{i} A_{i}\right)^{1 / 2} .
$$

In order to check the accuracy of equation (5) and the lower bound (equation (6)), we have made exact calculations of the energy of simple 2D clusters. The clusters that we calculated consist of a central cell with $n$ sides symmetrically surrounded by one or two shells of cells, as in figure 1. The clusters are bidisperse, with $N_{1}$ bubbles of area $A_{1}$ and $N_{2}$ bubbles of area $A_{2}$. We calculated clusters of this type with the bubble arrangements indicated in figure 1 , and for various $n$ and various ratios $\lambda=A_{2} / A_{1}$, with $\lambda<1$. Note that, for each topology, the areas 1 and $\lambda$ can be interchanged. More complicated asymmetrical clusters are difficult to solve analytically. Similar calculations, but for monodisperse symmetrical 2D clusters $(\lambda=1)$, were previously made by Vaz and Fortes (2001).

For a given topology, the clusters of figure 1 are solved by imposing the Plateau laws (circular films meeting at $120^{\circ}$ at triple junctions with zero sum of the curvatures) and the areas of the bubbles. This gives a number of equations equal to the number of unknown variables (Fortes and Teixeira 2002). In general, there is one and only one solution to the $n$-fold symmetrical clusters, but in some cases there is no solution (e.g. the cluster in figure $1(b)$ with $n=5$ and with inner cells $\lambda=0.5$ and external cells 1 , or the cluster in figure $1(\mathrm{~g})$ with $n=3$ and $\lambda=0.05)$. We did not consider eventual solutions without $n$-fold symmetry (Weaire et al. 2002). We solved one-shell clusters with $3 \leqslant n \leqslant 9$ and $\lambda=0.05,0.25,0.33$ and 0.5 and two-shell clusters with $3 \leqslant n \leqslant 7$ and the same values of $\lambda$. The perimeter calculations were made on the same line as for monodisperse symmetrical clusters (Vaz and Fortes 2001). The total number of clusters calculated was 120 .

We have compared the calculated values of the total perimeter $E / \gamma$ with equations (5) and (6). Figure 2 shows the calculated data points, including values

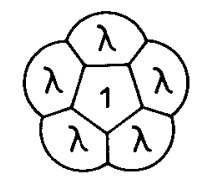

(a)

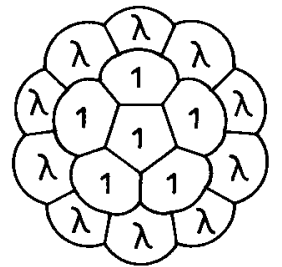

(b)

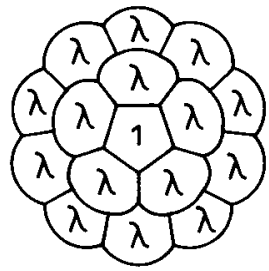

(e)

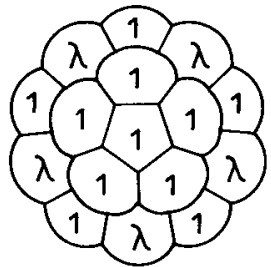

(c)

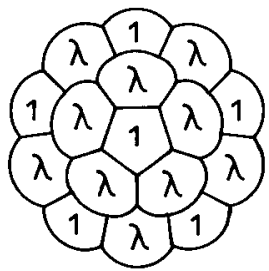

(f)

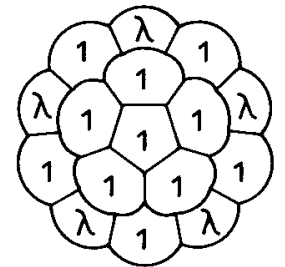

(d)

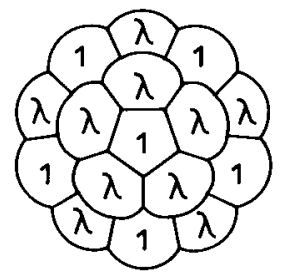

(g)

Figure 1. 2D clusters for which we made exact calculations: $(a)$ a central cell of area 1 surrounded by one shell of cells of area $\lambda ;(b)-(g)$ a central cell of area 1 surrounded by two shells of cells of areas 1 and $\lambda$. Note that 1 and $\lambda$ can be interchanged. 


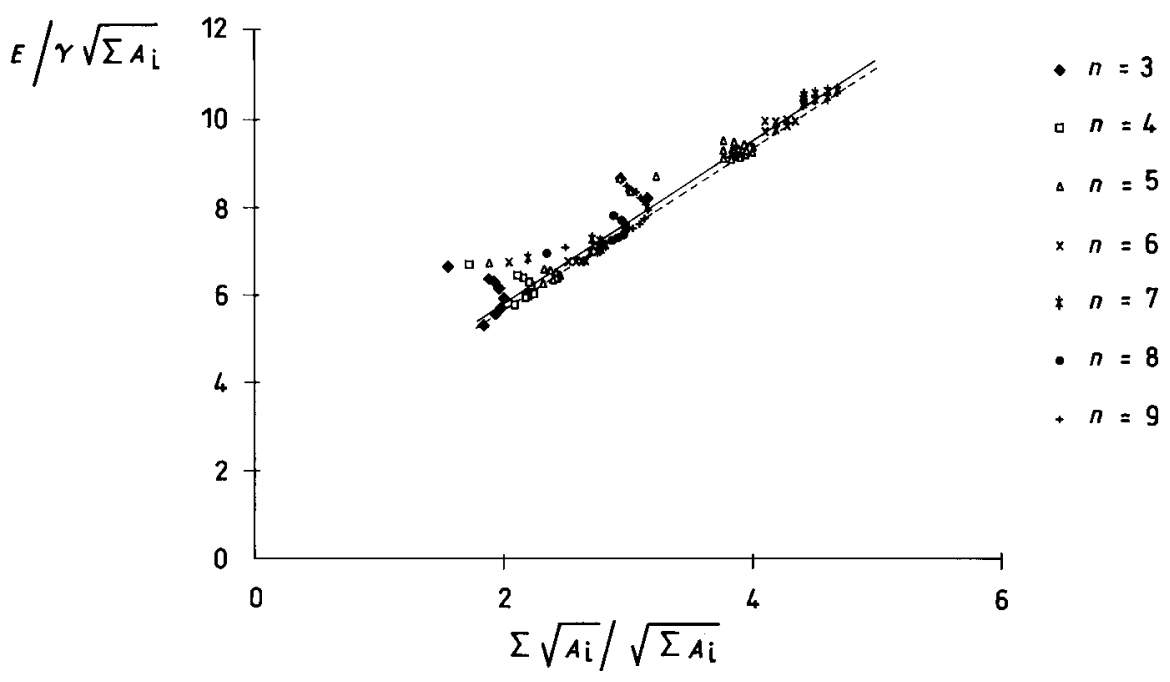

Figure 2. $E / \gamma\left(\sum_{i} A_{i}\right)^{1 / 2}$ as a function of $\sum_{i} A_{i}^{1 / 2} /\left(\sum_{i} A_{i}\right)^{1 / 2}$, where the calculated values for various clusters are indicated ( $n$ is the number of sides of the central cell; see figure 1). $(-)$, equation (5) (the points that deviate most from equation (5) are for the lower value of $\lambda(=0.05)$ used in the calculations). ( ---$)$, lower bound given by equation (6).

for $\lambda=1$ from the calculations of Vaz and Fortes (2001), and plots of equations (5) and (6) (solid and broken straight lines respectively) with $E / \gamma\left(\sum_{i} A_{i}\right)^{1 / 2}$ as a function of $\sum_{i} A_{i}^{1 / 2} /\left(\sum_{i} A_{i}\right)^{1 / 2}$. As anticipated, deviations from equation (5) occur for the lower values of $\lambda$, particularly for the lower $n$. For example, for $\lambda=0.05$ and $n=3$, the energy calculated from equation (5) is $25 \%$ lower than the actual energy. However, for $\lambda>0.2$ and $n \geqslant 4$, the error is always below $8 \%$.

On the other hand, some clusters in figure 2 have energies below the conjectured lower bound (6). This can be attributed to the small number of bubbles in those clusters.

If the clusters are not equiaxed, equation (4) should be replaced by

$$
s_{\mathrm{b}}=2 \varepsilon\left(\pi \sum_{i} A_{i}\right)^{1 / 2},
$$

where $\varepsilon$ is a shape factor defined as the ratio of the cluster perimeter to that of a circle of area equal to the total area of the cluster. For a round cluster, $\varepsilon=1$; for other clusters, $\varepsilon>1$. The general approximate equation that we propose for the energy $E / \gamma$ of a $2 \mathrm{D}$ cluster is then

$$
\frac{E}{\gamma}=\frac{3.722}{2} \sum_{i} A_{i}^{1 / 2}+2.042 \varepsilon\left(\sum_{i} A_{i}\right)^{1 / 2} .
$$

Again, $E / \gamma$ obtained from this equation should be regarded as a reasonable approximation; positive deviations will increase as the width of the distributions of area and of number of sides increase.

It is tempting to suggest an equation similar to equation (8) for 3D bubble clusters, based on equivalent assumptions. The minimum surface area partition of $3 \mathrm{D}$ space into regions of equal volume is the Weaire-Phelan tiling, for which the 
surface area per unit volume is 5.288 (Weaire and Phelan 1994, Weaire and Hutzler 1999). The figure for the Kelvin tiling is about $0.3 \%$ larger (Kraynik and Reinelt, 1996, Weaire and Hutzler 1999). The areas for bubbles of other topologies are not known, but we expect, by analogy with the $2 \mathrm{D}$ problem, that they will be close to 5.288 per unit volume. The external boundary specific energy $\gamma_{\mathrm{b}}$ of a 3D cluster of Kelvin bubbles was estimated by Fortes and Rosa (2001). They found for the average $\gamma_{\mathrm{b}}$ the value

$$
\gamma_{\mathrm{b}}=0.586 \gamma
$$

We assume that this $\gamma_{\mathrm{b}}$ applies to arbitrary 3D clusters. The surface area of the external boundary of a spherical cluster of volume $\sum_{i} V_{i}$ is $4 \pi\left[(3 / 4 \pi) \sum_{i} V_{i}\right]^{2 / 3}$. The equation that we propose for the surface energy of a $3 \mathrm{D}$ free cluster with bubbles of volume $V_{i}$ is

$$
\frac{E}{\gamma}=\frac{5.288}{2} \sum_{i} V_{i}^{2 / 3}+2.833 \varepsilon\left(\sum_{i} V_{i}\right)^{2 / 3},
$$

where $\varepsilon$ is a shape factor defined as the ratio of the area of the cluster external boundary to the area of a sphere of volume equal to that of the cluster. The accuracy of this equation can, in principle, be checked with analytical or numerical calculations by, for example, using the Surface Evolver program (Brakke 1992).

\section{REFERENCES}

Brakke, K., 1992, Exp. Math., 1, 141.

Fortes, M. A., and Rosa, M. E., 2001, J. Colloid Interface Sci., 241, 205.

Fortes, M. A., and Teixeira, P. I. C., 2002, Eur. Phys. J. E, 6, 255.

Graner, F., Jiang, Y., Janiaud, E., and Flament, C., 2001, Phys. Rev. E, 63, 11402.

Hales, T. C., 2001, Discrete comput. Geom., 25, 1.

Kraynik, A. M., and Reinelt, D. A., 1996, J. Colloid Interface Sci., 181, 511.

Teixeira, P. I. C., Graner, F., and Fortes, M. A., 2002, Phys. Rev. E (to be published).

Vaz, M. F., and Fortes, M. A., 2001, J. Phys.: condens. Matter, 13, 1395.

Weaire, D., Cox, S., and Graner, F., 2002, Eur. Phys. J. E, 7, 123.

Weaire, D., and Hutzler, S., 1999, The Physics of Foams (Oxford: Clarendon).

Weaire, D., and Phelan, R., 1994, Phil. Mag. Lett., 69, 107. 